\title{
Research on Order Decision of Quantity Flexibility Contract under the Competition between Multiple Suppliers and Multiple Retailers
}

\author{
Cheng Qian', Yanan Tao', Zhilin Qin 1,2* \\ ${ }^{1}$ School of Science, Nantong University, Nantong, China \\ ${ }^{2}$ Nantong Vocational University, Nantong, China \\ Email: *qin.zhl@ntu.edu.cn
}

How to cite this paper: Qian, C., Tao, Y.N. and Qin, Z.L. (2018) Research on Order Decision of Quantity Flexibility Contract under the Competition between Multiple Suppliers and Multiple Retailers. American Journal of Industrial and Business Management, 8, 1879-1892.

https://doi.org/10.4236/ajibm.2018.89127

Received: July 3, 2018

Accepted: September 1, 2018

Published: September 4, 2018

Copyright $\odot 2018$ by authors and Scientific Research Publishing Inc. This work is licensed under the Creative Commons Attribution International License (CC BY 4.0).

http://creativecommons.org/licenses/by/4.0/

\begin{abstract}
In the supply chain system consisting of multiple suppliers and multiple retailers, in order to realize supply chain coordination, we studied the ordering decision under the quantity flexibility contract with competition between both sides. On the one hand, from the perspective of suppliers, a satisfactory production of goods for each supplier was obtained by means of a kind of interactive programmer step method. On the other hand, from the perspective of retailers, because each supplier holds a different preference attitude for different retailers, we sort retailers as preference in application of a group decision sequencing method of Markov chain. The optimal order quantity of goods for each retailer was obtained at the same time and we prove that it is Pareto optimal. Finally, we give a numerical example.
\end{abstract}

\section{Keywords}

Supplier, Retailer, Competition, Quantity Flexibility, Order Decision

\section{Introduction}

Optimal supply chain performance requires the execution of a precise set of actions [1]. Unfortunately, the lack of mutual constraints among the members of the supply chain makes the decentralized supply chain operation inefficient. However, optimal performance is achievable if the firms coordinate by signing contracts. Cachon studied six types of contracts including wholesale price contract, buyback contract, revenue sharing contract, quantity flexibility contract, 
sales rebate contract and quantity discount contract [1]. Currently, the information sharing system between the upstream and downstream enterprises in the supply chain is imperfect, and information asymmetry appears. The imbalance between the quantity of commodity production and the market demand forecast leads to the oversupply of goods or the shortage of supply. It is possible to avoid overproduction or out-of-stock by signing quantity flexibility contracts. Quantity flexibility contract allows the retailer to adjust the initial order quantity after the partial or full resolution of demand uncertainty, which helps the retailer reduce supply-demand mismatches.

In the market supply chain, the supplier formulates a production plan based on the order quantity of the retailer and the production cost of the unit product. On the contrary, the retailer determines the order quantity of the goods based on the unit wholesale price and market demand forecast. This research will study order decision of multiple suppliers and multiple retailers under the quantity flexibility. In the suppliers competition model, we achieve a satisfactory production of goods for each supplier by means of a kind of interactive programmer step method. Then we prove that the solution obtained by this method is preferred by all suppliers, and it is also the weakly efficient solution. In the retailers competition model, because each supplier holds a different preference attitude for different retailers, we sort retailers as preference in application of a group decision sequencing method of Markov chain. At the same time we get the optimal order quantity of goods for each retailer and prove that the order quantity through a sequencing method in group decision making based on Markov chain is Pareto optimal. This provides basis for production and ordering decisions of supply chain members.

The article will be organized as follows: We begin with an introduction of our research in Section 1. The literature review is in Section 2. In Section 3, we introduce two models: Suppliers competition model and retailers competition model. In Section 4, we apply our models in a real case. Section 5 summarizes the paper and identifies areas for future research.

\section{Literature Review}

Many scholars have done research on quantity flexibility contracts. Chopra et al. proved that the flexibility quantity contract may be more effective than the return decision if the return cost is high [2]. Wu [3] considered a decentralized supply chain with a supplier and a retailer. Under a quantity flexibility contract retailer used Bayesian procedure to update demand information, and maked ultimate purchase commitment, which is constrained by the negotiated flexibility and the supplier's production. By numerical analysis it was shown that given other parameters fixed, more flexibility always benefits the retailer, while the supplier can only benefit from very small quantity flexibility. Lian et al. [4] explored a class of supply contracts under which a buyer receives discounts for committing to purchases in advance. As time rolls forward, the buyer can in- 
crease the order quantities for future periods of the rolling horizon based on updated demand forecast information and inventory status. They developed a finite-horizon dynamic programming model to characterize the structure of the optimal replenishment strategy for the buyer and present heuristic approaches to calculate the order volume in each period of the rolling horizon. Kesen et al. [5] considered the case where the buyer releases a fixed period replenishment order to the supplier under a supply contract defined by three parameters. A key buyer decision then is quantity lost $(\mathrm{QL})$, the order or replenishment quantity level below which no order is placed and the sales are lost. A model for deriving the expected supply and lost sales cost as a function of QL is presented, and it is shown that the optimal value of QL is the inflexion point of the lost sales cost and the quantity penalty. Mahajan [6] considered a quantity flexibility contract in a supply chain under price dependent demand. They showed that if the wholesale price lies in a certain range, there is a positive buyback fraction that the supplier would prefer. Through numerical work they found that the contract results in a win-win situation for both the supplier and the retailer in the case of price dependent demand. Kim [7] studied a bilateral contract with order quantity flexibility. To conduct comparative simulations, he developed four-echelon supply chain models, that employ the contracts and different forecasting techniques under dynamic market demands. The simulation outcomes showed that demand fluctuation can be effectively absorbed by the contract scheme, which enables better inventory management and customer service. Bicer and Hagspiel [8] considered a quantity flexibility contract with one supplier and one retailer. They used the multiplicative martingale model of forecast evolution to analyze the impact of lead-time reduction on the value of quantity flexibility for the retailer and found that the shorter the lead time, the higher the value of quantity flexibility. Li and Lian studied a quantity-flexibility contract with supply-chain coordination in the cosmetic industry. They developed a two-period dynamic model and obtain an optimal replenishment strategy for the retailer and the optimal pricing scheme for the supplier. By comparing it between models with and without quantity adjustment, the numeric alanalys is and their case application supported their theoretical model assertion that the synergy of attaining global (channel) optimal profit for both the supplier and the retailer is feasible. The results also showed that the advantages of the quantity-flexibility contract with the supply-chain coordination is very significant [9]. Karakaya and Bakal analyzed a decentralized supply chain with a single retailer and a single supplier where the retailer sells multiple products in a single period. The supplier has two options for procurement. The first procurement option is regular delivery at the beginning of the period, after the initial orders of the retailer. The next one is expedited delivery, after the updated orders are received. In this setting, they characterized the optimal policies for the retailer and the supplier, and assessed the benefits of flexibility [10]. Shi and Chen analyzed a decentralized supply chain consisting of a supplier and a retailer and proved that there always exist quanti- 
ty-flexibility contracts that coordinate the supply chain under the objective of expected profit maximization and are simultaneously Pareto optimal for the satisficing objective [11]. Based on Shi's research, Wang [12] studied the supply chain system with multiple retailers, and proved that the multi-retailer flexibility contract is Pareto optimal under multi targets with expected profit. Kim and Park studied quantity flexibility contract with two or more heterogeneous suppliers. From the buyer's perspective they developed a linear programming model including several key features of a quantity flexibility contract. A rolling-horizon implementation strategy has been suggested for efficient implementation of the contract. By numerical analysis, it was shown that the proposed method can be used to determine a cost effective solution for the buyer in a reasonable amount of time [13].

Most scholars focus on the research of quantity flexibility contract mainly around single supplier and single retailer or multi suppliers and single retailer. This article mainly discusses the ordering decisions of multiple suppliers and multiple retailers under quantity flexibility contracts, and analyzes the profit optimization under the multiple suppliers competition model and the multiple retailers competition model.

\section{Model and Analysis}

\subsection{Mathematical Notations}

Let $D$ be demand during the selling season. Let $F$ be the distribution function of demand and $f$ its density function: $F$ is differentiable, strictly increasing and $F(0)=0$. The production and sales of goods are completed by $m$ suppliers and $n$ retailers. The delivery cost of a unit of the product of supplier $s_{i}(i=1,2, \cdots, m)$ is $c_{i} . w_{i}$ is the wholesale price of a unit of the product offered by supplier $s_{i}$. The retailer $r_{j}^{\prime} s(j=1,2, \cdots, n)$ demand is $D_{j}$ and $D=\sum_{j=1}^{n} D_{j}$. The retail price for retailer $r_{j}$ is $p_{j}$ and the order quantity is $q_{j}$. The retailer earns $v$ per unit unsold at the end of season. Let $v<c_{i}<w_{i}<p_{j}$. The order matrix between suppliers and retailers is as follows:

$$
\begin{aligned}
& \begin{array}{lllll}
r_{1} & r_{2} & \cdots & r_{n}
\end{array}
\end{aligned}
$$

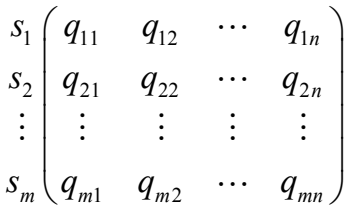

The quantity of products which the supplier $s_{i}$ supplies to the retailer $r_{j}$ is $q_{i j}$. For convenience, $x_{i}=\sum_{j=1}^{n} q_{i j}$ represents the total amount of product provided by supplier $s_{i}$. Similarly $q_{j}=\sum_{i=1}^{m} q_{i j}$ means the retailer $r_{j}^{\prime} s$ total order quantity from suppliers. According to the initial order quantity of the retailer, 
$\left[\alpha_{i} q_{i j}, \beta_{i} q_{i j}\right]$ is the ordering interval of each retailer given by each supplier in quantity flexibility contract, and $0 \leq \alpha_{i} \leq 1,1 \leq \beta_{i} \leq 2$. That is, the minimum order quantity of the retailer $r_{j}$ from the supplier $s_{i}$ should not be lower than $\alpha_{i} q_{i j}$ and retailer $r_{j}$ cannot exceed the maximum order quantity $\beta_{i} q_{i j}$ from supplier $s_{i} \cdot \bar{x}_{i}$ stands for the maximum production of the supplier $s_{i}$ and $\underline{x}_{i}$ is the minimal production.

\subsection{Suppliers Competition Model}

Total expected order quantity for supplier $s_{i}(i=1,2, \cdots, m)$ is

$$
\begin{aligned}
Q_{i} & =\alpha_{i} x_{i} \int_{-\infty}^{\alpha_{i} x_{i}} f(y) \mathrm{d} y+\int_{\alpha_{i} x_{i}}^{\beta_{i} x_{i}} y f(y) \mathrm{d} y+\beta_{i} x_{i}\left(1-\int_{-\infty}^{\beta_{i} x_{i}} f(y) \mathrm{d} y\right) \\
& =\beta_{i} x_{i}-\int_{\alpha_{i} x_{i}}^{\beta_{i} x_{i}} F(y) \mathrm{d} y
\end{aligned}
$$

Successively, the profit function is

$$
\begin{aligned}
\pi_{s_{i}}(\boldsymbol{x}) & =Q_{i} \cdot w_{i}+\left(\beta_{i} x_{i}-Q_{i}\right) \cdot v-\beta_{i} x_{i} \cdot c_{i} \\
& =w_{i}\left(\beta_{i} x_{i}-\int_{\alpha_{i} x_{i}}^{\beta_{i} x_{i}} F(y) \mathrm{d} y\right)+v \int_{\alpha_{i} x_{i}}^{\beta_{i} x_{i}} F(y) \mathrm{d} y-c_{i} \beta_{i} x_{i}
\end{aligned}
$$

In the supplier competition model, each supplier sets its own wholesale price and aims at its maximum profit. Through signing quantity flexibility contracts, the following multi-objective models can be established:

$$
\left\{\begin{array}{l}
\max \left(\pi_{s_{1}}(\boldsymbol{x}), \pi_{s_{2}}(\boldsymbol{x}), \cdots, \pi_{s_{m}}(\boldsymbol{x})\right) \\
\text { s.t. } \boldsymbol{x} \in L
\end{array}\right.
$$

where $L=\left\{\boldsymbol{x}=\left(x_{1}, \cdots, x_{m}\right)^{\mathrm{T}} \in R^{m} \mid \underline{x}_{i} \leq x_{i} \leq \bar{x}_{i}, \sum_{i=1}^{m} x_{i}=D, i=1,2, \cdots, m\right\} \quad$ is constraint set.

Under the circumstance that each supplier pursues their own profits the most, the suppliers with high wholesale prices may suffer losses due to excessive production of products. Therefore, in order to avoid the blind decision among the suppliers and improve the expected benefit of each supplier, the suppliers can know each other's production capacity through information sharing, then they determine the production of their respective commodities. Since the order quantity of a product is a flexible interval, a kind of interactive programmer called step method (STEM) can be used to determine the optimal product quantity for each supplier [14].

Step one: Solve the minimum and maximum of each objective function:

$\underline{\pi}_{s_{i}}$ denotes the minimum of each objective function and $\bar{\pi}_{s_{i}}$ is the maximum of each objective function. $\overline{\boldsymbol{x}}_{i}$ is the optimal solution of problem $\max _{\boldsymbol{x} \in X} \pi_{s_{i}}\left(\boldsymbol{x}_{i}\right)$.

Step two: Check each maximum point

- If $\overline{\boldsymbol{x}}=\overline{\boldsymbol{x}}_{1}=\overline{\boldsymbol{x}}_{2}=\cdots=\overline{\boldsymbol{x}}_{m}$, then $\overline{\boldsymbol{x}}$ is the optimal solution to problem.

- If $\overline{\boldsymbol{x}}_{1}, \overline{\boldsymbol{x}}_{2}, \cdots, \overline{\boldsymbol{x}}_{m}$ are not all the same, proceed to the next step. 
Step three: Normalize objective function

Select the normalized interval $[0, b](b>0)$, let

$$
\pi_{s_{i}}^{*}(\boldsymbol{x})=\frac{b}{\bar{\pi}_{s_{i}}-\underline{\pi}_{s_{i}}}\left(\pi_{s_{i}}(\boldsymbol{x})-\underline{\pi}_{s_{i}}\right)(i=1,2, \cdots, m)
$$

then get normalized comprehensive objective function:

$$
\pi_{s}^{*}(\boldsymbol{x})=\left(\pi_{s_{1}}^{*}(\boldsymbol{x}), \pi_{s_{2}}^{*}(\boldsymbol{x}), \cdots, \pi_{s_{m}}^{*}(\boldsymbol{x})\right)^{\mathrm{T}}
$$

Step four: Calculate the problem of the initial ideal target point.

Give the weight coefficient $u_{i}\left(u_{i}>0, \sum_{i=1}^{m} u_{i}=1\right)$ of importance of each objective function $\pi_{s_{i}}\left(x_{i}\right)$ approaching the ideal target value $\bar{\pi}_{s_{i}}$. Let $\lambda=\min _{1 \leq i \leq m} u_{i} \pi_{s_{i}}^{*}(\boldsymbol{x})$, then solve auxiliary problems:

$$
\left\{\begin{array}{l}
\max \lambda \\
\text { s.t. } u_{i} \pi_{s_{i}}^{*}(\boldsymbol{x}) \geq \lambda(i=1,2, \cdots, m) \\
\boldsymbol{x} \in L, \lambda \geq 0
\end{array}\right.
$$

Get a set of optimal solutions $\left(\boldsymbol{x}^{a \mathrm{~T}}, \lambda^{a}\right)^{\mathrm{T}}$, write $a=1$, and $\boldsymbol{x}^{1}=\left(x_{1}^{1}, x_{2}^{1}, \cdots, x_{m}^{1}\right)^{\mathrm{T}}$. Step five: Judge satisfaction

The supplier compares the current target value $\left(\pi_{s_{1}}\left(\boldsymbol{x}^{1}\right), \pi_{s_{2}}\left(\boldsymbol{x}^{1}\right), \cdots, \pi_{s_{m}}\left(\boldsymbol{x}^{1}\right)\right)^{\mathrm{T}}$ with the ideal target value $\left(\bar{\pi}_{s_{1}}, \bar{\pi}_{s_{2}}, \cdots, \bar{\pi}_{s_{m}}\right)^{\mathrm{T}}$ and the anti-ideal target value $\left(\underline{\pi}_{s_{1}}, \underline{\pi}_{s_{2}}, \cdots, \underline{\pi}_{s_{m}}\right)^{\mathrm{T}}$.

- If all suppliers are satisfied with the current goal, the solution is $\left(x_{1}^{1}, x_{2}^{1}, \cdots, x_{m}^{1}\right)^{\mathrm{T}}$.

- If none of the suppliers are satisfied with the current goals, there is no satisfactory solution.

- If $l(l<m)$ suppliers are satisfied with the current goals $\left(\pi_{s_{1}}\left(\boldsymbol{x}^{1}\right), \pi_{s_{2}}\left(\boldsymbol{x}^{1}\right), \cdots, \pi_{s_{l}}\left(\boldsymbol{x}^{1}\right)\right)^{\mathrm{T}}$ and $m-l$ suppliers are not satisfied with the current goals $\left(\pi_{s_{l+1}}\left(\boldsymbol{x}^{1}\right), \pi_{s_{l+2}}\left(\boldsymbol{x}^{1}\right), \cdots, \pi_{s_{m}}\left(\boldsymbol{x}^{1}\right)\right)^{\mathrm{T}}$, proceed to the next step. Step six: Give a satisfactory target tolerance constraint set, normalize the unsatisfied target weight coefficient.

- For the current satisfactory target $\left(\pi_{s_{1}}\left(\boldsymbol{x}^{1}\right), \pi_{s_{2}}\left(\boldsymbol{x}^{1}\right), \cdots, \pi_{s_{l}}\left(\boldsymbol{x}^{1}\right)\right)^{\mathrm{T}}$, the corresponding tolerance $\Delta \delta_{i} \geq 0(i=1,2, \cdots, l)$ is given as tolerance constraint set:

$$
L^{\prime}=\left\{\boldsymbol{x} \in L \mid \pi_{s_{i}}(\boldsymbol{x}) \geq \pi_{s_{i}}\left(\boldsymbol{x}^{1}\right)-\Delta \delta_{i}, i=1,2, \cdots, l\right\}
$$

- Let $v_{i}=\pi_{s_{i}}\left(\boldsymbol{x}^{1}\right)-\underline{\pi}_{s_{i}}(i=l+1, l+2, \cdots, m)$. Normalize dissatisfied goals and give their corresponding weights:

$$
u_{i}^{\prime}=\frac{v_{i}}{\sum_{i=l+1}^{m} v_{i}}(i=l+1, l+2, \cdots, m) \text {. }
$$

Step seven: Solve numerical optimization problem: 


$$
\left\{\begin{array}{l}
\max \lambda \\
\text { s.t. } u_{i}^{\prime} \pi_{s_{i}}^{*}(\boldsymbol{x}) \geq \lambda(i=l+1, l+2, \cdots, m) \\
\boldsymbol{x} \in L^{\prime}, \lambda \geq 0
\end{array}\right.
$$

We can get the optimal solution $\left(\boldsymbol{x}^{a+1 \mathrm{~T}}, \lambda^{a+1}\right)^{\mathrm{T}}$, then set $a=a+1$. If all suppliers are satisfied with the solution, problem (2) is solved, otherwise turn to step five.

This is repeated until a set of commodity production quantities satisfying each supplier is obtained, so that each supplier obtains the optimal profit. Finally, we get the solution $\left(\tilde{\boldsymbol{x}}^{\mathrm{T}}, \tilde{\lambda}\right)^{\mathrm{T}}$.

In the process of solving the problem (2), no matter problem (5) or (6), it is an ideal target point problem with a maximum coordinate distance as an approximation distance. As a result, the following conclusion can be obtained:

Theorem 3.1. The ideal evaluation function is constructed by weighting the maximum of the normalized objective function:

$$
g\left(\pi_{s}^{*}(\boldsymbol{x})\right)=\min _{1 \leq i \leq m} u_{i} \pi_{s_{i}}^{*}(\boldsymbol{x})
$$

If $g\left(\pi_{s}^{*}(\boldsymbol{x})\right)=\min _{1 \leq i \leq m} u_{i} \pi_{s_{i}}^{*}(\boldsymbol{x})$ is a increasing function of $\pi_{s}^{*}$, then the solution $\left(x_{1}^{a}, x_{2}^{a}, \cdots, x_{m}^{a}\right)^{\mathrm{T}}$ obtained in the fourth step is the weakly efficient solution of the problem (2). Therefore the final solution $\left(\tilde{x}_{1}, \tilde{x}_{2}, \cdots, \tilde{x}_{m}\right)^{\mathrm{T}}$ is the solution preferred by all suppliers, and it is also the weakly efficient solution of the problem (2).

Proof: $\forall \boldsymbol{x}^{\prime}, \boldsymbol{x}^{\prime \prime} \in L$, when $\pi_{S}^{*}\left(\boldsymbol{x}^{\prime}\right)>\pi_{s}^{*}\left(\boldsymbol{x}^{\prime \prime}\right)$, there is

$$
g\left(\pi_{s}^{*}\left(\boldsymbol{x}^{\prime}\right)\right)=\min _{1 \leq i \leq m} u_{i} \pi_{s_{i}}^{*}\left(\boldsymbol{x}^{\prime}\right)>\min _{1 \leq i \leq m} u_{i} \pi_{s_{i}}^{*}\left(\boldsymbol{x}^{\prime \prime}\right)=g\left(\pi_{s}^{*}\left(\boldsymbol{x}^{\prime \prime}\right)\right),
$$

so $g\left(\pi_{s}^{*}(\boldsymbol{x})\right)$ is the increasing function of $\pi_{s}^{*}$.

In the third step of the iterative process of step method, if $\overline{\boldsymbol{x}}_{1}=\overline{\boldsymbol{x}}_{2}=\cdots=\overline{\boldsymbol{x}}_{m}$, it can be obtained from Equation (3):

$$
\pi_{s_{i}}(\boldsymbol{x})=\underline{\pi}_{s_{i}}+\frac{\bar{\pi}_{s_{i}}-\underline{\pi}_{s_{i}}}{b} \pi_{s_{i}}^{*}(\boldsymbol{x})(i=1,2, \cdots, m)
$$

then we can get

$$
\pi_{s_{i}}\left(\boldsymbol{x}_{i}\right)=\underline{\pi}_{s_{i}}+\frac{\bar{\pi}_{s_{i}}-\underline{\pi}_{s_{i}}}{b} \pi_{s_{i}}^{*}(\overline{\boldsymbol{x}}) \geq \underline{\pi}_{s_{i}}+\frac{\bar{\pi}_{s_{i}}-\underline{\pi}_{s_{i}}}{b} \pi_{s_{i}}^{*}(\boldsymbol{x})=\pi_{s_{i}}(\boldsymbol{x}) \quad \forall \boldsymbol{x} \in L .
$$

Therefore, it is the absolute optimal solution of the problem (2), which is the retailer's preferred solution.

If $\overline{\boldsymbol{x}}_{1}, \overline{\boldsymbol{x}}_{2}, \cdots, \overline{\boldsymbol{x}}_{m}$ not all the same, then $\boldsymbol{x}^{a}=\left(x_{1}^{a}, x_{2}^{a}, \cdots, x_{m}^{a}\right)^{\mathrm{T}}$ is the absolute optimal solution of the problem (5). So there is

$$
g\left(\pi_{s}^{*}\left(\boldsymbol{x}^{a}\right)\right)=\min _{1 \leq i \leq m} u_{i} \pi_{s_{i}}^{*}\left(\boldsymbol{x}^{a}\right)>\min _{1 \leq i \leq m} u_{i} \pi_{s_{i}}^{*}(\boldsymbol{x})=g\left(\pi_{s}^{*}(\boldsymbol{x})\right) \quad \forall \boldsymbol{x} \in L
$$

Let's suppose $\left(x_{1}^{a}, x_{2}^{a}, \cdots, x_{m}^{a}\right)^{\mathrm{T}}$ is not a weakly efficient solution of problem (2), then it is known from the weak efficient solution that there is $\hat{\boldsymbol{x}}$ belongs to $L$, so that 


$$
\pi_{s_{i}}(\hat{\boldsymbol{x}})>\pi_{s_{i}}\left(\boldsymbol{x}^{a}\right)(i=1,2, \cdots, m)
$$

Then from the Formula (5) we can get

$$
\pi_{s_{i}}^{*}(\hat{\boldsymbol{x}})>\pi_{s_{i}}^{*}\left(\boldsymbol{x}^{a}\right)(i=1,2, \cdots, m)
$$

SO

$$
\begin{aligned}
\pi_{s}^{*}(\hat{\boldsymbol{x}}) & =\left(\pi_{s_{1}}^{*}\left(\hat{x}_{1}\right), \pi_{s_{2}}^{*}\left(\hat{x}_{2}\right), \cdots, \pi_{s_{m}}^{*}\left(\hat{x}_{m}\right)\right)^{\mathrm{T}} \\
& >\left(\pi_{s_{1}}^{*}\left(x_{1}^{a}\right), \pi_{s_{2}}^{*}\left(x_{2}^{a}\right), \cdots, \pi_{s_{m}}^{*}\left(x_{m}^{a}\right)\right)^{\mathrm{T}}=\pi_{s}^{*}\left(\boldsymbol{x}^{a}\right)
\end{aligned}
$$

Because $g\left(\pi_{s}^{*}(\boldsymbol{x})\right)$ is an increasing function of $\pi_{s}^{*}$, we can obtain

$$
g\left(\pi_{s}^{*}(\hat{\boldsymbol{x}})\right)>g\left(\pi_{s}^{*}\left(\boldsymbol{x}^{a}\right)\right) .
$$

Obviously, the above formula contradicts Formula (7).

\subsection{Retailers Competition Model}

In retailer competition mode, each retailer set the sales price of commodity and pursue their respective profits the most. Through signing flexibility contracts, the profit function of retailers $r_{j}(j=1,2, \cdots, n)$ is

$$
\pi_{r_{j}}=\sum_{i=1}^{m}\left[p_{j}\left(\beta_{i} q_{i j}-\int_{0}^{\beta_{i} q_{i j}} F(y) \mathrm{d} y\right)+v \int_{0}^{\alpha_{i} q_{i j}} F(y) \mathrm{d} y-w_{i}\left(\beta_{i} q_{i j}-\int_{\alpha_{i} q_{i j}}^{\beta_{i} q_{i j}} F(y) \mathrm{d} y\right)\right]
$$

Due to differences in sales capacity, order quantity, product promotion, business reputation, and other factors of each retailer, each supplier has different levels of preference strength for each retailer. Because of the uncertainty of the number of suppliers and the number of retailers, the optimal order quantity of each retailer is obtained through a sequence method in group decision making based on Markov chain [15].

- When $m=n$, the order quantity of $n$ retailers is $\left(q_{1}, q_{2}, \cdots, q_{n}\right)$, the preference matrix is

$$
A_{1}=\left(\begin{array}{cccc}
\frac{q_{11}^{a}}{x_{1}^{a}} & \frac{q_{12}^{a}}{x_{1}^{a}} & \ldots & \frac{q_{1 n}^{a}}{x_{1}^{a}} \\
\frac{q_{21}^{a}}{x_{2}^{a}} & \frac{q_{22}^{a}}{x_{2}^{a}} & \cdots & \frac{q_{2 n}^{a}}{x_{2}^{a}} \\
\vdots & \vdots & \ddots & \vdots \\
\frac{q_{m 1}^{a}}{x_{m}^{a}} & \frac{q_{m 2}^{a}}{x_{m}^{a}} & \cdots & \frac{q_{m n}^{a}}{x_{m}^{a}}
\end{array}\right) .
$$

- When $m>n$, introduce $m-n$ virtual retailers, let

$$
\frac{q_{i j}^{a}}{x_{i}^{a}}=\left\{\begin{array}{l}
(1-\delta) \frac{q_{i j}^{a}}{x_{i}^{a}}, i=1,2, \cdots, m ; j=1,2, \cdots, n \\
\frac{\delta}{m-n}, i=1,2, \cdots, m ; j=n+1, n+2, \cdots, m
\end{array},\right.
$$

where $\delta \in(0,1)$, the preference matrix is 


$$
A_{2}=\left(\begin{array}{ccccccc}
(1-\delta) \frac{q_{11}^{a}}{x_{1}^{a}} & (1-\delta) \frac{q_{12}^{a}}{x_{1}^{a}} & \cdots & (1-\delta) \frac{q_{1 n}^{a}}{x_{1}^{a}} & \frac{\delta}{m-n} & \cdots & \frac{\delta}{m-n} \\
(1-\delta) \frac{q_{21}^{a}}{x_{2}^{a}} & (1-\delta) \frac{q_{22}^{a}}{x_{2}^{a}} & \cdots & (1-\delta) \frac{q_{2 n}^{a}}{x_{1}^{a}} & \frac{\delta}{m-n} & \cdots & \frac{\delta}{m-n} \\
\vdots & \vdots & \ddots & \vdots & \vdots & \ddots & \vdots \\
(1-\delta) \frac{q_{m 1}^{a}}{x_{m}^{a}} & (1-\delta) \frac{q_{m 2}^{a}}{x_{m}^{a}} & \cdots & (1-\delta) \frac{q_{m n}^{a}}{x_{m}^{a}} & \frac{\delta}{m-n} & \cdots & \frac{\delta}{m-n}
\end{array}\right) .
$$

- When $m<n$, Introduce $n-m$ virtual suppliers, let

$$
\frac{q_{i j}^{a}}{x_{i}^{a}}=\left\{\begin{array}{l}
\frac{q_{i j}^{a}}{x_{i}^{a}}, \quad i=1,2, \cdots, m ; j=1,2, \cdots, n \\
\frac{1}{n}, \quad i=m+1, m+2, \cdots, n ; j=1,2, \cdots, n
\end{array},\right.
$$

the preference matrix is

$$
A_{3}=\left(\begin{array}{cccc}
\frac{q_{11}^{a}}{x_{1}^{a}} & \frac{q_{12}^{a}}{x_{1}^{a}} & \ldots & \frac{q_{1 n}^{a}}{x_{1}^{a}} \\
\frac{q_{21}^{a}}{x_{2}^{a}} & \frac{q_{22}^{a}}{x_{2}^{a}} & \ldots & \frac{q_{2 n}^{a}}{x_{2}^{a}} \\
\vdots & \vdots & \ddots & \vdots \\
\frac{q_{m 1}^{a}}{x_{m}^{a}} & \frac{q_{m 2}^{a}}{x_{m}^{a}} & \ldots & \frac{q_{m n}^{a}}{x_{m}^{a}} \\
\frac{1}{n} & \frac{1}{n} & \ldots & \frac{1}{n} \\
\vdots & \vdots & \ddots & \vdots \\
\frac{1}{n} & \frac{1}{n} & \ldots & \frac{1}{n}
\end{array}\right) .
$$

In the matrix $A_{1}, A_{2}, A_{3}$, there are $0 \leq \frac{q_{i j}^{a}}{x_{i}^{a}} \leq 1(j=1,2, \cdots, n)$,

$\sum_{j=1}^{n} \frac{q_{i j}^{a}}{x_{i}^{a}}=1(i=1,2, \cdots, m)$. So the matrix $A_{1}, A_{2}, A_{3}$ is Markov matrix, then the equations

$$
\begin{aligned}
& \left(\lambda_{1}, \lambda_{2}, \cdots, \lambda_{n}\right) A_{1}=\left(\lambda_{1}, \lambda_{2}, \cdots, \lambda_{n}\right) \\
& \left(\lambda_{1}, \lambda_{2}, \cdots, \lambda_{n}\right) A_{2}=\left(\lambda_{1}, \lambda_{2}, \cdots, \lambda_{n}\right) . \\
& \left(\lambda_{1}, \lambda_{2}, \cdots, \lambda_{n}\right) A_{3}=\left(\lambda_{1}, \lambda_{2}, \cdots, \lambda_{n}\right)
\end{aligned}
$$

has unique solutions, where $0 \leq \lambda_{j}^{*} \leq 1$ and $\sum_{j=1}^{n} \lambda_{j}^{*}=1$. So the supplier's preference for retailer $r_{j}(j=1,2, \cdots, n)$ is $\lambda_{j}^{*}$, and retailer $r_{j}(j=1,2, \cdots, n)$ optimal order amount is $q_{j}^{*}=\lambda_{j}^{*} x^{a}$.

Theorem 3.2. Let $\left(\lambda_{1}^{*}, \lambda_{2}^{*}, \cdots, \lambda_{n}^{*}\right)$ be the solution of the equation

$$
\left(\lambda_{1}, \lambda_{2}, \cdots, \lambda_{n}\right) A_{1}=\left(\lambda_{1}, \lambda_{2}, \cdots, \lambda_{n}\right)
$$

or 


$$
\left(\lambda_{1}, \lambda_{2}, \cdots, \lambda_{n}\right) A_{2}=\left(\lambda_{1}, \lambda_{2}, \cdots, \lambda_{n}\right)
$$

or

$$
\left(\lambda_{1}, \lambda_{2}, \cdots, \lambda_{n}\right) A_{3}=\left(\lambda_{1}, \lambda_{2}, \cdots, \lambda_{n}\right)
$$

under

$$
0 \leq \frac{q_{i j}^{a}}{x_{i}^{a}} \leq 1(j=1,2, \cdots, n), \sum_{j=1}^{n} \frac{q_{i j}^{a}}{x_{i}^{a}}=1(i=1,2, \cdots, m) .
$$

Suppose $\frac{q_{i_{0} t}^{a}}{x_{i}^{a}} \geq \frac{q_{i_{0} j}^{a}}{x_{i}^{a}}(t, j=1,2, \cdots, m ; t \neq j)$, then $\lambda_{t}^{*} \geq \lambda_{j}^{*}$.

If $i_{0} \in\{1,2, \cdots, m\}$ exists so that

$$
\frac{q_{i_{0} t}^{a}}{x_{i}^{a}}>\frac{q_{i_{0} j}^{a}}{x_{i}^{a}}(t, j=1,2, \cdots, m ; t \neq j),
$$

then

$$
\lambda_{t}^{*}>\lambda_{j}^{*}
$$

Proof: When $m=n$, from

$$
\left(\lambda_{1}, \lambda_{2}, \cdots, \lambda_{n}\right) A_{1}=\left(\lambda_{1}, \lambda_{2}, \cdots, \lambda_{n}\right)
$$

we get

$$
\lambda_{t}^{*}-\lambda_{j}^{*}=\sum_{i=1}^{n}\left(\frac{q_{i t}^{a}}{x_{i}^{a}}-\frac{q_{i j}^{a}}{x_{i}^{a}}\right) \lambda_{i}^{*} \geq 0,
$$

so

$$
\lambda_{t}^{*} \geq \lambda_{j}^{*}
$$

If $\frac{q_{i_{0} t}^{a}}{x_{i}^{a}}>\frac{q_{i_{0} j}^{a}}{x_{i}^{a}}$, there is

$$
\lambda_{t}^{*}-\lambda_{j}^{*}=\sum_{\substack{i=1 \\ i \neq i_{0}}}^{n}\left(\frac{q_{i t}^{a}}{x_{i}^{a}}-\frac{q_{i j}^{a}}{x_{i}^{a}}\right) \lambda_{i}^{*}+\left(\frac{q_{i_{0} t}^{a}}{x_{i}^{a}}-\frac{q_{i_{0} j}^{a}}{x_{i}^{a}}\right) \lambda_{i}^{*}>0 .
$$

When $m>n$, from

$$
\left(\lambda_{1}, \lambda_{2}, \cdots, \lambda_{n}\right) A_{2}=\left(\lambda_{1}, \lambda_{2}, \cdots, \lambda_{n}\right)
$$

we get

$$
\begin{aligned}
\lambda_{t}^{*}-\lambda_{j}^{*} & =\sum_{i=1}^{m}\left(\frac{q_{i t}^{a}}{x_{i}^{a}}-\frac{q_{i j}^{a}}{x_{i}^{a}}\right) \lambda_{i}^{*} \\
& =\sum_{i=1}^{n}\left((1-\delta) \frac{q_{i t}^{a}}{x_{i}^{a}}-(1-\delta) \frac{q_{i j}^{a}}{x_{i}^{a}}\right) \lambda_{i}^{*}+\sum_{i=n+1}^{m}\left(\frac{\delta}{m-n}-\frac{\delta}{m-n}\right) \lambda_{i}^{*} \geq 0
\end{aligned}
$$

so

$$
\lambda_{t}^{*} \geq \lambda_{j}^{*}
$$

If $\frac{q_{i_{0} t}^{a}}{x_{i}^{a}}>\frac{q_{i_{0} j}^{a}}{x_{i}^{a}}$, there is 


$$
\begin{aligned}
\lambda_{t}^{*}-\lambda_{j}^{*}= & \sum_{i=1}^{m}\left(\frac{q_{i t}^{a}}{x_{i}^{a}}-\frac{q_{i j}^{a}}{x_{i}^{a}}\right) \lambda_{i}^{*} \\
= & \sum_{\substack{i=1 \\
i \neq i_{0}}}^{n}\left((1-\delta) \frac{q_{i t}^{a}}{x_{i}^{a}}-(1-\delta) \frac{q_{i j}^{a}}{x_{i}^{a}}\right) \lambda_{i}^{*}+\sum_{i=n+1}^{m}\left(\frac{\delta}{m-n}-\frac{\delta}{m-n}\right) \lambda_{i}^{*} \\
& +\left((1-\delta) \frac{q_{i_{0} t}^{a}}{x_{i}^{a}}-(1-\delta) \frac{q_{i_{0} j}^{a}}{x_{i}^{a}}\right) \lambda_{i}^{*}>0
\end{aligned}
$$

When $m<n$, from

$$
\left(\lambda_{1}, \lambda_{2}, \cdots, \lambda_{n}\right) A_{3}=\left(\lambda_{1}, \lambda_{2}, \cdots, \lambda_{n}\right)
$$

we get

$$
\lambda_{t}^{*}-\lambda_{j}^{*}=\sum_{i=1}^{n}\left(\frac{q_{i t}^{a}}{x_{i}^{a}}-\frac{q_{i j}^{a}}{x_{i}^{a}}\right) \lambda_{i}^{*}=\sum_{i=1}^{m}\left(\frac{q_{i t}^{a}}{x_{i}^{a}}-\frac{q_{i j}^{a}}{x_{i}^{a}}\right) \lambda_{i}^{*}+\sum_{i=m+1}^{n}\left(\frac{1}{n}-\frac{1}{n}\right) \lambda_{i}^{*} \geq 0,
$$

so

$$
\lambda_{t}^{*} \geq \lambda_{j}^{*}
$$

If $\frac{q_{i_{0} t}^{a}}{x_{i}^{a}}>\frac{q_{i_{0} j}^{a}}{x_{i}^{a}}$, there is

$$
\begin{aligned}
\lambda_{t}^{*}-\lambda_{j}^{*} & =\sum_{i=1}^{n}\left(\frac{q_{i t}^{a}}{x_{i}^{a}}-\frac{q_{i j}^{a}}{x_{i}^{a}}\right) \lambda_{i}^{*} \\
& =\sum_{\substack{i=1 \\
i \neq i_{0}}}^{m}\left(\frac{q_{i t}^{a}}{x_{i}^{a}}-\frac{q_{i j}^{a}}{x_{i}^{a}}\right) \lambda_{i}^{*}+\sum_{i=m+1}^{n}\left(\frac{1}{n}-\frac{1}{n}\right) \lambda_{i}^{*}+\left(\frac{q_{i_{0} t}^{a}}{x_{i}^{a}}-\frac{q_{i_{0} j}^{a}}{x_{i}^{a}}\right) \lambda_{i}^{*}>0
\end{aligned}
$$

All of the above three cases meet $\lambda_{t}^{*}>\lambda_{j}^{*}$.

Theorem 3.2 shows that the retailer order quantity through a sequencing method in group decision making based on Markov chain is Pareto optimal.

\section{Numerical Analysis}

The following gives an example for the supplier competition model.

This is a simplifying assumption for otherwise our numerical work will have to be very extensive. We suppose there are three suppliers that produce the same product. The market demand is set to 100 and the supplier's production capacity interval is [20,60]. Market demand meets condition

$$
f(y)=\left\{\begin{array}{lc}
\frac{1}{100}, & 0 \leq y \leq 100 \\
0, & \text { others }
\end{array} .\right.
$$

Other parameters are shown in Table 1.

With matlab program, we get the results as follows (Table 2).

Then we assume that suppliers $s_{1}, s_{2}$ are not satisfied with their profits and supplier $s_{3}$ is satisfied with profit. Supplier $s_{3}$ can reduce the profit to 102.40 . The results are shown in Table 3. 
Table 1. Parameter value.

\begin{tabular}{cccccccc}
\hline Notations & value & Notations & value & Notations & value & Notations & value \\
\hline$w_{1}$ & 10 & $c_{2}$ & 5 & $\beta_{1}$ & 1.2 & $\beta_{3}$ & 1.1 \\
$w_{2}$ & 9 & $c_{2}$ & 5 & $\alpha_{2}$ & 0.9 & $u_{1}$ & 0.4 \\
$w_{3}$ & 8 & $V$ & 2 & $\beta_{2}$ & 1.2 & $u_{2}$ & 0.3 \\
$c_{1}$ & 4 & $\alpha_{1}$ & 0.7 & $\alpha_{3}$ & 0.9 & $u_{3}$ & 0.3 \\
\hline
\end{tabular}

Table 2. Operation results.

\begin{tabular}{cccc}
\hline suppliers & $S_{1}$ & $S_{2}$ & $S_{3}$ \\
\hline output & 30.1 & 34.5 & 35.4 \\
profit & 182.29 & 139.35 & 112.40 \\
\hline
\end{tabular}

Table 3. Operation results after adjusting once.

\begin{tabular}{cccc}
\hline suppliers & $S_{1}$ & $s_{3}$ & $S_{3}$ \\
\hline output & 33.5 & 34.6 & 31.9 \\
profit & 198.55 & 139.68 & 102.63
\end{tabular}

We assume that supplier $s_{2}$ is not satisfied with its profit and suppliers $s_{1}, s_{3}$ are satisfied with profit. Supplier $s_{1}$ can reduce the profit to 193.55. We get results as follows (Table 4).

The following is to find the optimal order quantity of the retailers through Markov chain.

We suppose there are 3 suppliers and 3 retailers. In order to simplify the calculation, the supply of each supplier according to the above is $33,35,32$ respectively. We suppose the preference matrix between suppliers and retailers is

$$
A_{1}=\left(\begin{array}{ccc}
\frac{10}{33} & \frac{12}{33} & \frac{11}{33} \\
\frac{11}{35} & \frac{9}{35} & \frac{15}{35} \\
\frac{9}{32} & \frac{11}{32} & \frac{12}{32}
\end{array}\right) .
$$

By solving equation

$$
\left(\lambda_{1}, \lambda_{2}, \lambda_{3}\right) A_{1}=\left(\lambda_{1}, \lambda_{2}, \lambda_{3}\right)
$$

we can get

$$
\left(\lambda_{1}, \lambda_{2}, \lambda_{3}\right) \approx(0.298,0.322,0.380) .
$$

At this time, the optimal order quantity of the goods of each retailer is

$$
q_{1}=29.8, q_{2}=32.2, q_{3}=38.0 \text {. }
$$

Through numerical analysis, when $\frac{q_{i_{0} t}^{a}}{x_{i}^{a}}>\frac{q_{i_{0} j}^{a}}{x_{i}^{a}}(t, j=1,2, \cdots, m ; t \neq j)$, the 
Table 4. Operation results after adjusting twice.

\begin{tabular}{cccc}
\hline suppliers & $S_{1}$ & $S_{3}$ & $s_{3}$ \\
\hline output & 32.5 & 35.6 & 31.9 \\
profit & 193.86 & 142.93 & 102.63 \\
\hline
\end{tabular}

condition is $\lambda_{t}^{*}>\lambda_{j}^{*}$ satisfied. This confirms that the solution we obtained by this method is Pareto optimal.

\section{Conclusion}

This article establishes profit models of suppliers and retailers under quantity flexibility contracts respectively in the case of the multi-suppliers and multi-retailers competition. According to each supplier setting the quantity of goods as the flexibility interval, through step method we obtain the production quantity of all suppliers which make the suppliers in the competitive state get the optimal profit. This is the production volume satisfied by all suppliers, which not only avoids the blind production decision of each supplier, but also ensures the supply of the upstream supply chain to meet the market demand. Secondly, we use a sequence method in group decision making based on Markov chain to rank each retailer's preferences and determine the optimal order quantity for each retailer. This helps retailers to make reasonable orders and avoid excessive inventory. The article does not consider the specific ordering quantity of each retailer to each supplier, which needs further research.

\section{Conflicts of Interest}

The authors declare no conflicts of interest regarding the publication of this paper.

\section{References}

[1] Cachon, G.P. (2003) Supply Chain Coordination with Contracts. Handbooks in Operations Research and Management Science, 11, 227-339.

[2] Chopra, S. and Meindl, P. (2001) Supply Chain Management: Strategy, Planning, and Operation. Prentice Hall, New Jersey.

[3] Wu, J. (2005) Quantity Flexibility Contracts under Bayesian Updating. Computers \& Operations Research, 32, 1267-1288.

[4] Lian, Z. and Deshmukh, A. (2009) Analysis of Supply Contracts with Quantity Flexibility. European Journal of Operational Research, 196, 526-533.

[5] Kesen, S.E., Kanchanapiboon, A. and Das, S.K. (2010) Evaluating Supply Chain Flexibility with Order Quantity Constraints and Lost Sales. International Journal of Production Economics, 126, 181-188.

[6] Mahajan, S. (2014) A Quantity Flexibility Contract in a Supply Chain with Price Dependent Demand. OPSEARCH, 51, 219-234. https://doi.org/10.1007/s12597-013-0137-2

[7] Kim, W.S. (2013) A Supply Chain Contract with Flexibility as a Risk-Sharing Mechanism for Demand Forecasting. International Journal of Systems Science, 44, 
1134-1149. https://doi.org/10.1080/00207721.2011.653592

[8] Bicer, I. and Hagspiel, V. (2016) Valuing Quantity Flexibility under Supply Chain Disintermediation Risk. International Journal of Production Economics, 180, 1-15.

[9] Li, X., Lian, Z., Choong, K.K. and Liu, X. (2016) A Quantity-Flexibility Contract with Coordination. International Journal of Production Economics, 179, 273-284.

[10] Karakaya, S. and Bakal, I.S. (2013) Joint Quantity Flexibility for Multiple Products in a Decentralized Supply Chain. Computers \& Industrial Engineering, 64, 696-707.

[11] Shi, C. and Chen, B. (2008) Pareto Quantity Flexibility Contracts for a Supply Chain under Multiple Objectives. Journal of the Operational Research Society, 59, 685-692. https://doi.org/10.1057/palgrave.jors.2602378

[12] Wang, J.C., Xie, Y. and Wang, W.Q. (2013) Pareto Optimal Quantity Flexibility Contracts in Multi-Objective Supply Chain. Journal of Nantong University, 12, 49-56.

[13] Kim, J.S., Park, S.I. and Shin, K.Y. (2014) A Quantity Flexibility Contract Model for a System with Heterogeneous Suppliers. Computers \& Operations Research, 41, 98-108.

[14] Teargny, T., Renayoun, R., de Montgolfier, J. and Larichev, O. (1971) Linear Programming with Multiobjective Functions Step Method (STEM). Mathematical Programming, 1, 366-375. https://doi.org/10.1007/BF01584098

[15] Qin, Z.L. and Hu, Y.D. (2000) A Sequencing Method in Group Decision-Making Based on Markov Chain. Journal of Guizhou University, 17, 1-12. 